\section{ADAPTATION OF THE MOHR VOLUMETRIC METHOD TO GENERAL DETERMINATIONS OF CHLORINE}

\author{
BY LESTER YODER \\ Received April 11, 1919
}

By the proper preliminary preparation of the chloride solutions many determinations of chlorine as usually carried out may be simplified and shortened by the use of Mohr's volumetric method. Other volumetric methods are less satisfactory as the following brief résumé will show:

The original Volhard method has been modified by Drechsel, ${ }^{1}$ Rothmund and Burgstaller, ${ }^{2}$ and Alefeld, ${ }^{3}$ to a short and accurate process. Excess of standardized silver nitrate solution is added to the chloride solution and the excess titrated with standardized thiocyanate. The precipitate of silver chloride must be rendered either more insoluble by flocculation with heat or reagents or filtered from the excess of silver nitrate for the titration with the thiocyanate.

Votocek ${ }^{4}$ recently reported a method in which the chlorides are titrated in about $250 \mathrm{cc}$. of slightly acid solution with mercuric nitrate solution using $0.06 \mathrm{~g}$. of sodium nitroprusside crystals as the indicator. In this method nitrites and sulfites interfere.

The well-known method of Mohr in which alkaline or alkaline-earth chlorides react with silver nitrate in the presence of a few drops of potassium chromate solution as indicator is as simple, direct, and fully as accurate as the method of Votocek, provided the proper conditions for the titration are as easily attained. The conditions necessary for an accurate titration appear numerous and exacting because (I) the solution must be neutral, (2) no carbonates or phosphates may be present since the silver salts of these acids are less soluble than the red silver chromate indicator and would therefore be titrated as well as the chlorides, and (3) the solution of the chlorides must have a proper concentration to produce the most definite color change.

The object of the work herein presented was to develop and test modifications of procedure to meet these conditions so that the Mohr method would be applicable to rapid determinations of chlorine in various materials and particularly in organic products containing small amounts of the element.

In the preparation of a titratable solution of the material in which chlorine is to be determined the sample must be ignited (in the case of organic products, at a low heat), and provision made for the fixation of free chlorine. An excess of sodium carbonate is usually added with the result that the sodium salts fuse and thereby prevent even after repeated ignitions, the complete oxidation of the carbonaceous matter which will pass through the filter in the subsequent filtration. A large amount of carbonates will also be present in the solution and the removal of any excess of phosphates is not assured.

Since the modifications necessary for the use of the

\footnotetext{
12 . anal. Chem., 16 (1877), 351.

Z. anorg. Chem., 63 (1909), 330

3. anal. Chem., 48 (1909), 79.

4 Chem.-Ztg., 42 (1918), 257-60.
}

Mohr titration must provide for the removal of carbonates and phosphates, calcium acetate solution was substituted for the solution of sodium carbonate and ferric acetate solution was subsequently added to the ignited residue.

The following procedure gave the best results:

To the sample is added $5 \mathrm{cc}$. of a 30 per cent solution of calcium acetate with sufficient distilled water to thoroughly saturate the material. The mixture is evaporated to dryness at $120^{\circ} \mathrm{C}$. and ignited at a temperature not over $450^{\circ} \mathrm{C}$. The cooled residue is thoroughly moistened with a few cubic centimeters of a ro per cent solution of ferric acetate, and again evaporated to dryness and ignited below $450^{\circ} \mathrm{C}$. The residue is triturated with hot water, filtered, and washed until free from chlorides. The filtrate is evaporated to dryness or nearly to dryness and taken up with just sufficient hot water to insure complete solution of the chlorides, when cooled. After the addition of 2 or 3 drops of potassium chromate, the solution is titrated with $0.05 N$ silver nitrate.

The calcium acetate fixes free chlorine and during the extraction of the ignited residue precipitates carbonates and neutralizes the filtrate for the titration. The ferric acetate precipitates the phosphates and is itself converted into the insoluble oxide and also removed by the one filtration. Two ignitions withorganic materials have not failed in our work to give clear and colorless solutions. Fair examples of the results obtained in the above procedure are reported in the table.

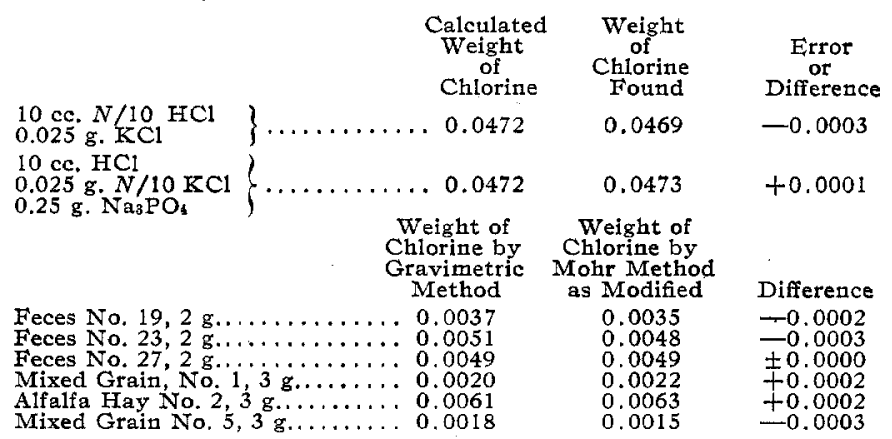

The modified method was applied to two solutions, one containing known amounts of free hydrochloric acid and its potassium salt, and another containing in addition added soluble phosphate. These were evaporated and ignited twice and subjected to a temperature of $450^{\circ} \mathrm{C}$. in one of the ignitions. The results show no loss of chlorine and only a negligible difference from the calculated weight of chlorine in the samples. Determinations of chlorine were also made in different: kinds of cow feces and mixed grain and in alfalfa hay. The results differ only slightly from those of determinations made gravimetrically and are well within the limits of experimental error although the percentage error in the weights of chlorine found is large by reason of the low chlorine content of the materials.

With the procedure outlined and from the results obtained by its use it seems that the Mohr direct titration method could be used for determinations of total chlorine in most instances.

\footnotetext{
Chemistry section

Iowa Agricultural, Experimeint Station AMes, Iowa
} 